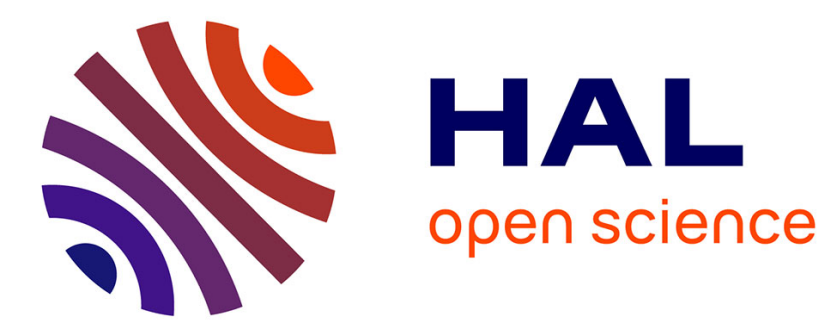

\title{
Cryostat pour études sous compression uniaxiale
}

B. Butowiez, J.P. Danoy

\section{- To cite this version:}

B. Butowiez, J.P. Danoy. Cryostat pour études sous compression uniaxiale. Revue de Physique Appliquée, 1969, 4 (1), pp.67-69. 10.1051/rphysap:019690040106700 . jpa-00242894

\section{HAL Id: jpa-00242894 https://hal.science/jpa-00242894}

Submitted on 1 Jan 1969

HAL is a multi-disciplinary open access archive for the deposit and dissemination of scientific research documents, whether they are published or not. The documents may come from teaching and research institutions in France or abroad, or from public or private research centers.
L'archive ouverte pluridisciplinaire HAL, est destinée au dépôt et à la diffusion de documents scientifiques de niveau recherche, publiés ou non, émanant des établissements d'enseignement et de recherche français ou étrangers, des laboratoires publics ou privés. 


\title{
GRYOSTAT POUR ÉTUDES SOUS GOMPRESSION UNIAXIALE
}

\author{
Par B. BUTOWIEZ et J. P. DANOY, \\ Laboratoire de Physique M.P. I, Faculté des Sciences, place Victor-Hugo, 13-Marseille.
}

(Reçu le 21 octobre 1968.)

\begin{abstract}
Résumé. - On décrit un cryostat permettant de faire des études optiques à 2 oK, le cristal étant soumis à une compression uniaxiale.
\end{abstract}

Abstract. - A cryostat is described which can be used for optical investigations down to $2 \mathrm{oK}$, the crystal being submitted to an uniaxial compression.

I. Introduction. - L'un des problèmes majeurs de la spectroscopie est la détermination des niveaux d'énergie d'un système quelconque. Cette détermination se fait par exemple à partir du spectre d'émission ou d'absorption du système et le premier problème auquel se heurte le spectroscopiste est le dépouillement et le classement des spectres. Dans le cas des solides, cette recherche est compliquée, car tous les constituants du cristal qui sont, dans ce cas, régulièrement répartis aux nœuds d'un réseau cristallin, réagissent les uns sur les autres, ce qui multiplie le nombre des niveaux d'énergie et donne naissance à une absorption plus ou moins continue.

Une autre cause de brouillage est due à l'agitation thermique et, pour obtenir une meilleure résolution des spectres, on la diminue autant que possible en opérant à basse température. Dans ces conditions, on observe souvent, dans les solides cristallisés, des spectres de raies fines d'émission ou d'absorption dont les longueurs d'onde sont aisément mesurables. C'est le cas par exemple pour les chromates et bichromates dont la bande d'absorption qui s'étend jusque dans le visible donne naissance du côté des grandes longueurs d'onde à un spectre de raies fines $[1,2]$.

Pour le classement de ces spectres, certaines méthodes expérimentales sont classiques et ont été essayées. En particulier, dans le cas du bichromate de potassium, l'application d'une induction magnétique de 25000 gauss en utilisant un spectrographe Huet type A II (dispersion $45 \AA / \mathrm{mm}$ autour de $5500 \AA$ ) n'a donné lieu à aucun effet observable. L'utilisation d'un spectrographe plus dispersif (spectrographe Jarrel-Ash type Ebert Mark IV, dispersion 2,4 $\AA / \mathrm{mm}$ ) ne pourra permettre, quel que soit le résultat, de résoudre totalement le problème, la structure vibrationnelle n'apparaissant plus à forte dispersion [3]

Il existe un autre procédé d'études qui semble, depuis une dizaine d'années, être de plus en plus employé et qui consiste à soumettre le cristal soit à une pression hydrostatique, soit à une contrainte uniaxiale. En effet, si l'on considère un cristal ionique par exemple, les ions sont régulièrement répartis aux nœuds du réseau et la pression a pour effet, en déformant le cristal, de modifier légèrement les distances interioniques. Il en résulte que les interactions entre ions sont légèrement modifiées et que les niveaux d'énergie du système sont déplacés. Ces déplacements entraînent une modification de la fréquence des raies et, dans le cas où elles sont fines, leur déplacement est très sensible à la moindre déformation du cristal. L'idée générale a donc été d'utiliser ce phénomène physique, en profitant de l'excellente résolution des spectres à basse température pour mettre en évidence des variations de la fréquence, donc de la longueur d'onde des raies d'absorption. L'étude de ces déplacements, dans la mesure où les fréquences vibrationnelles ne sont pas affectées par ce phénomène, permettra de classer ces raies en séries, une série étant constituée de la transition électronique pure et de la structure vibrationnelle qui lui est liée.

De nombreuses expériences ont été faites, en particulier par Drickamer et coll. [4] pour des pressions allant jusqu'à $250 \mathrm{~kb}$. Les appareils utilisés sont volumineux et ne permettent de faire des études qu'à des températures proches de l'ambiance [5]. De tels effets ont également été observés, soit sur des ions de terres rares, soit sur des centres colorés dans des halogénures alcalins $[6,7]$, et les cryostats utilisés dérivent en général de l'appareil de D. G. Thomas [8]. Cet appareil ne peut être utilisé dans le cas de cristaux minces où la contrainte doit être appliquée perpendiculairement à la grande face du cristal, c'est-à-dire parallèlement à la direction d'observation. Une bombe à glace $[9,10]$, utilisable à basse température et permettant de soumettre le cristal à une pression hydrostatique, a conduit à des résultats intéressants, mais il était nécessaire de faire une étude en compression uniaxiale afin de relier les déplacements observés 
aux contraintes appliquées. D'autre part, dans la mesure où une pression hydrostatique ne modifie pas la symétrie cristalline, elle ne permet pas de lever des dégénérescences et ne peut qu'accentuer celles qui sont déjà levées. Au contraire, une contrainte uniaxiale modifie la symétrie cristalline. Un cryostat a donc été conçu et l'idée directrice en est la suivante :

- L'appareil doit être utilisable à la température de l'hélium liquide et permettre des mesures à température encore inférieure par pompage sur le réfrigé-

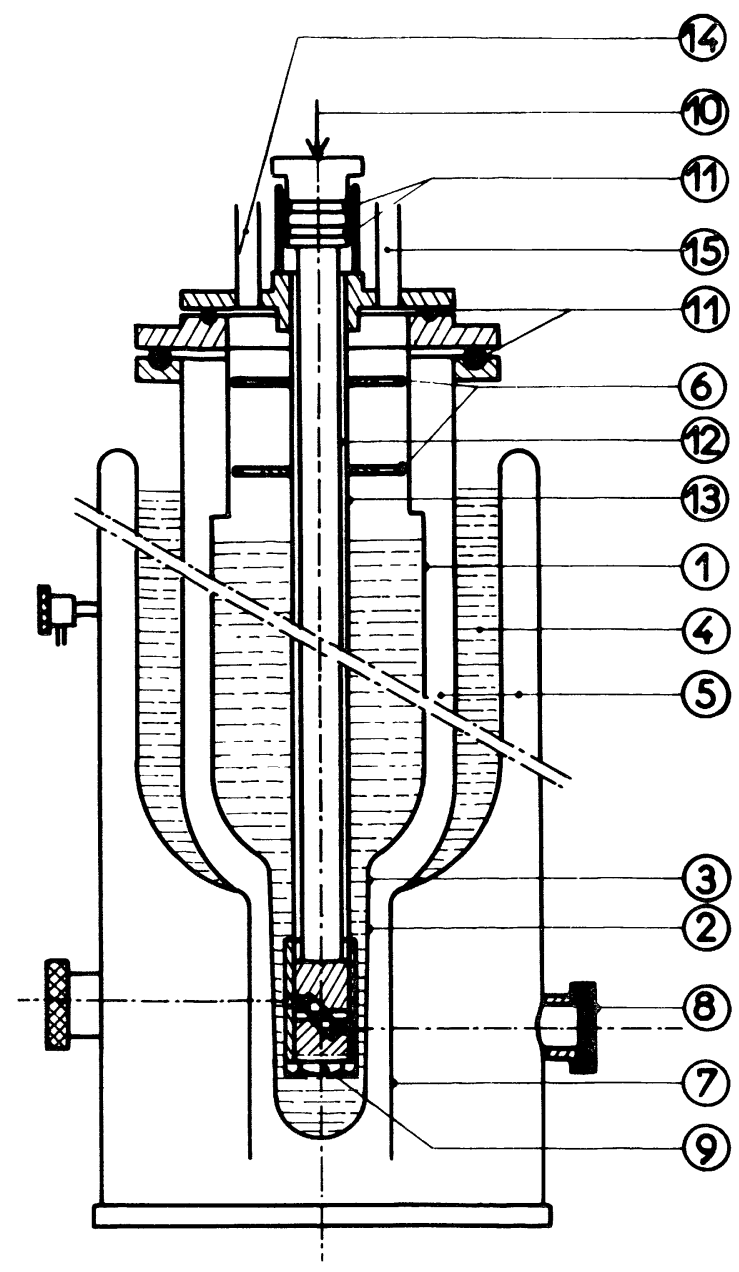

a rant. Le cristal étant refroidi en l'absence de toute contrainte, une compression uniaxiale variable et mesurable doit être appliquée perpendiculairement à la grande face du cristal. Enfin, le système doit être très lumineux, utilisable en ultraviolet éventuellement et être commode d'utilisation.

II. Réalisation. - Le cryostat représenté sur la figure 1 a dérive de l'appareil décrit par $P$. Pesteil et R. Philip [11]. La cellule haute pression constituant la partie optique est représentée sur la figure $1 \mathrm{~b}$. L'effort s'exerce sur le cristal par l'intermédiaire de deux prismes à réflexion totale en silice dont la face servant de miroir a été aluminée pour éviter les pertes éventuelles de lumière. Le prisme inférieur est fixé dans un bouchon se vissant dans un cylindre en aluminium. Le cristal est orienté et collé sur ce prisme, la partie non utile du prisme étant cachée soit à l'aide

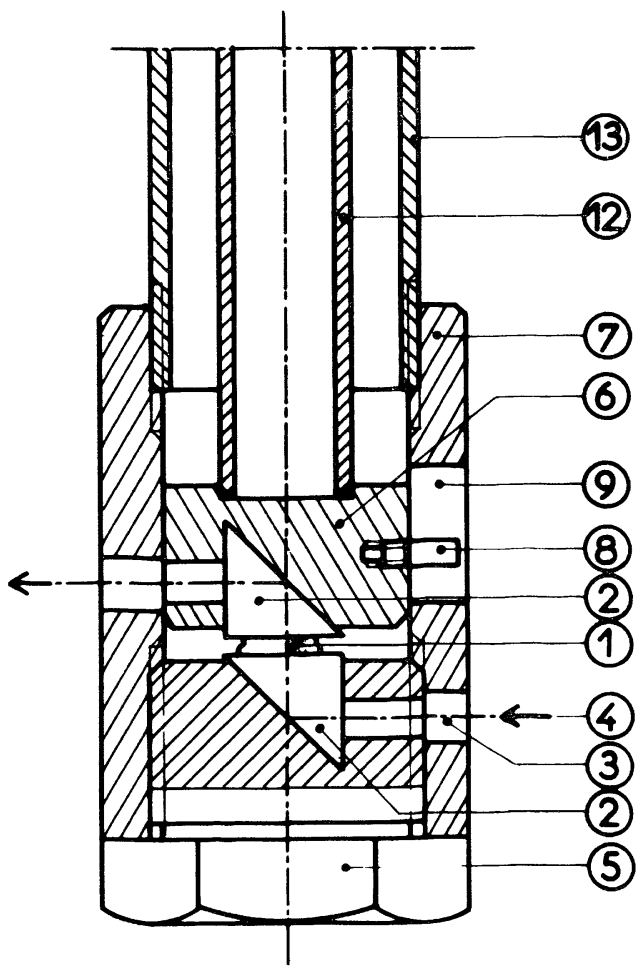

$\mathrm{b}$

FIG. 1.

a) Schéma général du cryostat pour études sous compression uniaxiale : (1), vase interne contenant le réfrigérant; (2), partie en silice; (3), joint Kovar-silice; (4), garde d'azote liquide ; (5), vide ; (6), écrans thermiques ; (7), écran thermique en cuivre percé de fenêtres faisant vis-à-vis de celles du cryostat (8) et de celles de la cellule haute pression; (9), cellule optique haute pression; (10), force appliquée; (11), joints toriques d'étanchéité. La force est transmise par le tube poussoir (12) coulissant dans le tube guide (13) ; orifices de remplissage (14) et d'évacuation (15).

b) Détail de la partie optique à haute pression : (1), cristal étudié ; $(2)$, prismes à réflexion totale en silice ; (3), fenêtre ; (4), trajet du faisceau lumineux; (5), bouchon portant le prisme inférieur fixe ; (6), piston portant le prisme supérieur mobile; (7), cylindre en aluminium; $(8)$, guide coulissant à frottement doux dans une rainure (9) parallèle à l'axe du cylindre (7). La force s'exerce sur le piston par le tube poussoir (12) coulissant dans le tube guide (13). 
d'une peinture, soit à l'aide d'un peu de vacoplast. Le piston portant le prisme supérieur est descendu dans le cylindre et une vis solidaire de ce piston coulisse à frottement doux dans une rainure taillée dans le cylindre selon une génératrice. Au cours du déplacement, l'arête du prisme supérieur est ainsi maintenue parallèle à celle du prisme inférieur fixe. Ge piston est doucement posé sur le cristal. Get ensemble est ensuite vissé à l'extrémité du tube guide solidaire de la platine supérieure qui est orientée et fixée sur le cryostat. Après purge de l'air intérieur, le tube poussoir est descendu dans le cryostat et posé sans peser sur le piston. Après prérefroidissement, on siphonne l'hélium dans le cryostat.

Cet appareil a été utilisé dans notre laboratoire, en particulier pour l'étude des spectres d'absorption des chromate et bichromate de potassium. Les monocristaux de surface $0,1 \mathrm{~cm}^{2}$ et d'épaisseurs allant de quelques dizaines de microns à $1 \mathrm{~mm}$ ont pu être soumis à des efforts variables de 0 à $100 \mathrm{ou} 120 \mathrm{kgf}$ sans qu'ils se cassent. Les manipulations ont été effectuées à des températures comprises entre 1,6 oK et 2,16 oK par pompage sur l'hélium, l'opération en milieu superfluide présentant en outre l'avantage d'augmenter considérablement la luminosité. Dans ces conditions, la durée d'utilisation qui était de 2 heures s'est toujours avérée amplement suffisante.

Les résultats relatifs à l'absorption du chromate de potassium et à l'effet de la pression ont été publiés [2]. Le spectre d'absorption débute par un triplet de raies fines attribué à la transition ${ }^{1} A_{1} \rightarrow{ }^{3} T_{1}$. La dégénérescence du niveau triplet ${ }^{3} T_{1}$ est partiellement levée par le champ cristallin, cette levée de dégénérescence étant accentuée par la contrainte appliquée au cristal. La position des raies d'absorption en fonction de la pression exercée est représentée sur la figure 2.

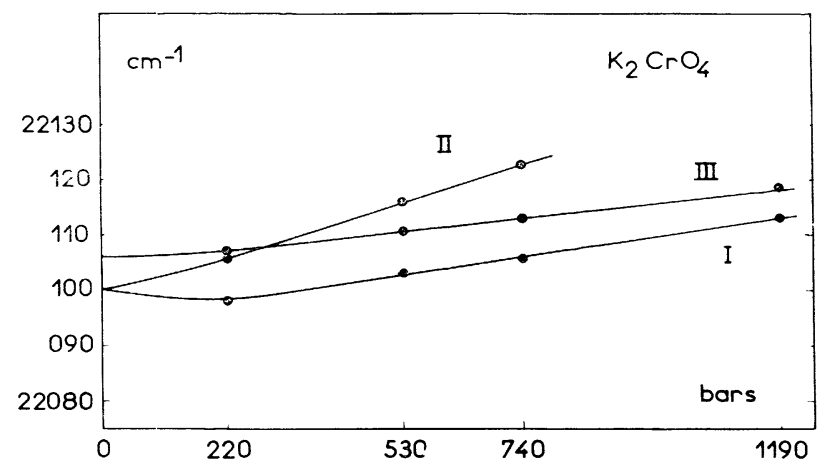

FIG. 2. - Spectre d'absorption de $\mathrm{K}_{2} \mathrm{CrO}_{4}$ à 2 oK. Influence d'une compression uniaxiale sur la position des raies de la transition électronique pure ${ }^{1} A_{1} \rightarrow^{3} T_{1}$. La lame monocristalline est taillée parallèlement à la face $q^{\prime}$ du cristal telle qu'elle est définie par P. Groth (Chemische Kristallographie).

III. Conclusion. - Cet appareil de construction simple est très commode d'utilisation. Il présente l'avantage d'être très lumineux et de pouvoir être utilisé avec des appareils très dispersifs. La manipulation la plus délicate consiste à appliquer l'effort uniformément sur le cristal, et la planéité et le parallélisme des faces de ce dernier doivent être travaillés avec soin.

\section{BIBLIOGRAPHIE}

[1] Teltow (J.), Z. Phys. Chem. (Leipzig), 1939, B 43 375.

Bu'Towiez (B.), C. R. Acad. Sc., 1967, 264, série B, 948 et 1212 .

[2] Butowiez (B.), C. R. Acad. Sc., 1968, 267, série B, 1234.

[3] Butowrez (B.), C. R. Acad. Sc., 1968, 266, série B, 1083.

[4] Drickamer (H. G.) et coll. Pour une revue complète, voir par exemple : High Pressure Physics and Chemistry, vol. I, Academic Press, chap. 5.VI. Solid State Physics, vol. 17, 1.

[5] Drickamer (H. G.) et coll., J. Opt. Soc. Am., 1957, 47, 1015 .
[6] KAPLIANSKII (A. A.), Optics and Spectroscopy, 1959, 7, 406, etc.

[7] VON DER OSTEN (W.), Z. Phys., 1964, 174, 423 ; voir également "Colloque sur les centres colorés ", Saclay, 16 mars 1967, supplément au Journal de Physique, fasc. 8-9, p. C4-72.

[8] Thomas (D. G.), J. Appl. Phys. (Suppl.), 1961, 32, 2298.

[9] Pesteil (P.) et coll., C. R. Acad. Sc., 1966, 263, série B, 199.

[10] Butowiez (B.), C. R. Acad. Sc., 1966, 263, série B, 1037.

[11] Pesteil, (P.) et Philip (R.), Cryogenics, 1960, 1, 1, 49. 